\title{
DESAFIOS E BARREIRAS À IMPLANTAÇÃO DA GESTÃO DO CONHECIMENTO EM ORGANIZAÇÕES
}

\author{
Victor Horta \\ Mestrando em Ciência da Informação - UFMG \\ Bibliotecário do Instituto Tecnológico da Aeronáutica (ITA) \\ victorgrayfox@gmail.com \\ Ricardo Rodrigues Barbosa \\ Doutor em Administração \\ Professor titular da ECI/PPGCI- UFMG \\ rrbarb@gmail.com
}

\section{Resumo}

\begin{abstract}
O presente estudo, através de uma revisão sistemática de literatura, se propôs analisar artigos científicos que abordam as dificuldades de implantação ou gerenciamento de programas de gestão do conhecimento. A pesquisa teve como objetivo, estudar as principais categorias de barreiras à implantação da gestão do conhecimento que foram relatadas pelos autores em suas pesquisas, a fim de verificar a existência de barreiras consideradas recorrentes a um contexto organizacional geral ou a existência de barreiras específicas, limitadas apenas ao ambiente analisado (estudos de casos). A pesquisa bibliográfica foi executada na base de dados do Portal de Periódicos Capes. No processo de recuperação dos artigos, o período de busca foi definido entre os anos de 2009 a 2016. Foram analisados um total de 44 artigos com o tema proposto para o estudo. Os resultados indicaram a concentração de uso de três categorias específicas (individual, organizacional e tecnológica). Foi possível identificar, que na sua grande maioria, as barreiras à gestão do conhecimento são consideradas recorrentes a um contexto organizacional geral. A falta de confiança, a falta de apoio dos gestores da alta administração e a falta de tecnologias que atendam às necessidades de compartilhamento foram consideradas as barreiras mais recorrentes.
\end{abstract}

Palavras-chave: Gestão do Conhecimento, Barreiras, Desafios, Implantação, Organizações.

\section{INTRODUÇÃO}

Estamos presenciando um contexto onde a informação é transmitida em alta velocidade e, como resultado, as organizações procuram alterar sua maneira de agir para se adequarem a esse novo ambiente organizacional (LOPES; STADLER; KOVALESKI, 2003).

Neste novo contexto, o desenvolvimento e execução de procedimentos e atividades nas organizações, geram diversos tipos de dados indicadores, informações técnicas/administrativas e conhecimentos que norteiam a cadeia de futuras e possíveis decisões.

Quando bem gerenciados, os dados, as informações e o conhecimento permitem que as decisões tomadas contribuam para a melhoria e eficiência dos processos organizacionais (CHOO, 2006).

A evolução da tecnologia proporcionou mudanças que elevam a importância do conhecimento, tornando-o um recurso fundamental (PROBST; RAUB; ROMHARDT, 2002) e valioso nas organizações, conduzindo novas possibilidades de trabalho, comunicação, inovando estruturas tecnológicas e modificando a forma de interação humana (CHIAVENATO, 2003). NONAKA; TAKEUCHI (2008) considera que o núcleo para inovação é a produção de conhecimento.

O conhecimento se torna um bem competitivo que deve ser nutrido, um fator importante para a força econômica de uma organização (SVETLANA, 2007), e o seu gerenciamento permite que as organizações 
prevejam a natureza e o potencial das mudanças no ambiente organizacional, bem como a adequação de suas decisões estratégicas (LIN; WU; YEN, 2012).

Levando em consideração a importância do conhecimento, seu gerenciamento se torna uma tarefa muito desafiadora (SVETLANA, 2007). Um deficiente compartilhamento do conhecimento pode levar a potenciais falhas nas organizações, resultando em atrasos no desenvolvimento de produtos e serviços, custos aumentados dos processos e clientes insatisfeitos (BARSON et al, 2000).

Para lidar com essa problemática de controle, criação e fluxo do conhecimento nas organizações, a gestão do conhecimento foi estruturada como uma disciplina que foca sistematicamente a informação e a construção do conhecimento, visando à otimização do desempenho de resposta da empresa no ambiente, desenvolvendo o conhecimento corporativo (TREHAN, 2005).

Porém, o preenchimento das lacunas existentes no processo de implantação de um sistema de gestão do conhecimento envolve desafios para as organizações que passam pelo rompimento de hábitos, crenças e valores incorporados, que refletem indiretamente sua cultura organizacional e de gestão (RUNTE, 2016).

Pode se considerar que em um contexto sem aprendizagem organizacional, os indivíduos e as organizações tendem a repetir velhas práticas de gestão, acarretando em alguns casos, um deficiente controle de processos e falhas na aplicação técnica da equipe disponível (GARVIN, 2000). A partir desse contexto, o estudo de experiências anteriores no processo de implantação de iniciativas de gestão, se configura como um recurso em potencial para mitigar os travamentos da aprendizagem organizacional.

O compartilhamento efetivo de conhecimento é considerado crucial para uma organização e facilita a extração dos benefícios de seus recursos existentes (KUKKO, 2013), porém, essa realidade envolve um ambiente organizacional apropriado, com o processo de construção desenvolvida através de estudos de práticas anteriores conhecidas, implantadas e avaliadas (BEM; PRADO; DELFINO, 2013).

Existem obstáculos e lacunas naturais que dificultam as atividades envolvidas na gestão do conhecimento (EIRIZ; SIMÕES; GONÇALVES, 2007) e esses desafios relacionados à sua implantação, metodologias e modelos práticos não são triviais (TERRA, 2010), sendo de extrema importância a identificação de possíveis obstáculos que alinham ao caminho do controle do conhecimento (KUKKO, 2013).

A identificação das barreiras e seu impacto no compartilhamento de conhecimento e no comportamento de inovação individual é uma área de pesquisa em potencial (YESIL; HIRLAK, 2013), e através dos seus resultados, fornece informações para a sensibilização dos gestores de todos os níveis da organização quanto à importância da liderança frente aos desafios de se tornar a aprendizagem através do conhecimento, uma realidade do dia-a-dia nas organizações (GARVIN, 2000).

Assim, compreender os processos de gestão do conhecimento focando nos possíveis fatores que limitam, que ocasionam falhas ou promovem dificuldade no seu gerenciamento, visa garantir uma melhor estratégia estrutural de implantação, norteia o gestor do conhecimento, previne o desperdício de recursos e promove tomadas de decisões mais consistentes.

Porém para esse fim, é necessário o compartilhamento de conhecimentos formalizados em procedimentos, processos e práticas de trabalho que apoiem o esforço para progresso organizacional (BARSON et al, 2000).

\section{METODOLOGIA}

Revisões sistemáticas são estudos de literatura com o objetivo de avaliar criticamente e sintetizar evidências de vários estudos sobre o mesmo tópico (GRANT; BROTH, 2009), que têm como fonte de dados as publicações científicas que relatam os resultados das pesquisas em primeira mão (GALVÃO; PEREIRA, 2014). Caracteriza-se, pelo o emprego de uma metodologia de pesquisa visando minimizar o enviesamento da literatura, na medida em que é feita uma seleção exaustiva dos textos divulgados sobre o tema escolhido (TRANFIELD; DENYER; SMART, 2003).

O estudo propõe analisar artigos científicos que abordam as dificuldades à implantação ou gerenciamento de programas de gestão do conhecimento através de uma revisão sistemática de literatura. A pesquisa bibliográfica foi executada na base de dados do Portal de Periódicos, da Coordenação de 
Aperfeiçoamento de Pessoal de Nível Superior (Capes). No processo de recuperação dos artigos, o período de busca foi definido entre os anos de 2009 a 2016. Os termos adotados para as estratégias de recuperação dos artigos no Portal Capes foram knowledge management e barriers. Apesar da simplicidade da escolha terminológica, a combinação dos termos knowledge management e barriers nos campos de buscadores apresentou os melhores resultados de busca, recuperando uma quantidade superior de artigos com informações adequadas para análise e composição da pesquisa.

Foram analisados um total de 44 artigos com o tema proposto para o estudo. $\mathrm{O}$ processo metodológico de recuperação e seleção foi conduzido em três fases. A primeira fase consistiu em duas estratégias de buscas e recuperou um total de 43 artigos. A segunda fase, selecionou através da leitura integral dos artigos, os documentos relevantes que se enquadravam com os objetivos da pesquisa. Dos 43 artigos recuperados na primeira fase, foram selecionados apenas 35 artigos relevantes. A terceira fase analisou as referências bibliográficas utilizadas pelos autores para o desenvolvimento dos 35 artigos selecionados na segunda fase, extraindo os documentos mais citados, a fim de enriquecer o conteúdo para análise da pesquisa. Foram extraídos um total de oito artigos na terceira fase.
A extração do conteúdo dos documentos selecionados teve como foco principal a identificação das barreiras no gerenciamento do conhecimento e como os autores organizaram essas barreiras evidenciadas, analisando se foram organizadas em categorias/classificações ou não.

As categorias, barreiras e fatores limitantes identificados, foram organizadas em quadros referenciais, facilitando a análise, a fim de verificar se as barreiras e desafios podem ser considerados recorrentes no contexto organizacional ou se limitam apenas a especificidade do ambiente estudado.

Para a análise da existência de recorrência ou não das barreiras, os artigos foram estudados tendo como base referencial o artigo de RIEGE (2005), Three-dozen knowledge-sharing barriers managers must consider. A escolha do artigo de Riege como base referencial, se justifica pela quantidade de citações utilizadas pelos autores dos artigos selecionados em suas pesquisas, sendo um total de 16 citações, demonstrando a importância do autor para a temática estudada.

$\mathrm{O}$ critério adotado para considerar se as barreiras e fatores limitantes encontrados foram recorrentes ou não foi baseado na quantidade de vezes que autores distintos as evidenciaram em suas pesquisas. Para a barreira ser considerada recorrente, ela devia ser referenciada no mínimo, pela quantidade de cinco autores.

Quadro 1 - Artigos recuperados na primeira fase e selecionados na segunda fase

\begin{tabular}{|c|c|c|c|c|c|}
\hline \multicolumn{2}{|r|}{ Título } & \multirow{2}{*}{\begin{tabular}{l}
\multicolumn{1}{c}{ Autores } \\
Vazquez; \\
Fournier; \\
Flores. \\
\end{tabular}} & \multirow{2}{*}{ Ano } & \multirow{2}{*}{$\begin{array}{c}\text { Busca } \\
1^{\text {o }} \\
\text { estratégia }\end{array}$} & \multirow{2}{*}{$\begin{array}{l}2^{\circ} \text { fase } \\
\text { SIM }\end{array}$} \\
\hline 1 & $\begin{array}{l}\text { Overcoming cultural barriers for innovation and } \\
\text { knowledge production }\end{array}$ & & & & \\
\hline 2 & $\begin{array}{l}\text { Uncovering cultural perceptions and barriers during } \\
\text { knowledge audit. }\end{array}$ & Levy et al. & 2010 & $\begin{array}{l}2^{\circ} \\
\text { estratégia }\end{array}$ & SIM \\
\hline 3 & $\begin{array}{l}\text { Developing strategies for overcoming barriers to knowledge } \\
\text { sharing based on conversational knowledge management: a } \\
\text { case study of a financial company. }\end{array}$ & $\begin{array}{l}\text { Hong; } \\
\text { Suh; } \\
\text { Koo. }\end{array}$ & 2011 & $\begin{array}{l}1^{\circ} \\
\text { estratégia }\end{array}$ & SIM \\
\hline 4 & Knowledge management assessment trends. & Mcgurn. & 2011 & $\begin{array}{c}1^{\circ} \\
\text { estratégia } \\
\end{array}$ & SIM \\
\hline 5 & $\begin{array}{l}\text { Lessons from the business sector for successful knowledge } \\
\text { management in health care: a systematic review }\end{array}$ & $\begin{array}{l}\text { Kothari et } \\
\text { al. }\end{array}$ & 2011 & $\begin{array}{l}1^{\circ} \\
\text { estratégia }\end{array}$ & SIM \\
\hline 6 & $\begin{array}{l}\text { Knowledge-sharing enablers and barriers in pharmaceutical } \\
\text { research and development }\end{array}$ & $\begin{array}{l}\text { Lilleoere; } \\
\text { Hansen. }\end{array}$ & 2011 & $\begin{array}{l}2^{\circ} \\
\text { estratégia }\end{array}$ & SIM \\
\hline
\end{tabular}


Artigos de Revisão

\begin{tabular}{|c|c|c|c|c|c|}
\hline 7 & $\begin{array}{l}\text { Overcoming learning barriers through } \\
\text { knowledge management }\end{array}$ & $\begin{array}{l}\text { Dror; } \\
\text { Makany; } \\
\text { Kemp. }\end{array}$ & 2011 & $\begin{array}{l}2^{\circ} \\
\text { estratégia }\end{array}$ & NÃO \\
\hline 8 & $\begin{array}{l}\text { Exploring barriers to knowledge flow at different knowledge } \\
\text { management maturity stages }\end{array}$ & $\begin{array}{l}\text { Lin; } \\
\text { Wu; } \\
\text { Yen. }\end{array}$ & 2012 & $\begin{array}{l}2^{\circ} \\
\text { estratégia }\end{array}$ & SIM \\
\hline 9 & $\begin{array}{l}\text { Facilitators and barriers in local emergency knowledge } \\
\text { management: communities of practice in inter-organizational } \\
\text { partnerships }\end{array}$ & Ndlela. & 2012 & $\begin{array}{l}2^{\circ} \\
\text { estratégia }\end{array}$ & SIM \\
\hline 10 & $\begin{array}{l}\text { Knowledge sharing barriers and effectiveness at a higher } \\
\text { education institution }\end{array}$ & $\begin{array}{l}\text { Khalil; } \\
\text { Shea. }\end{array}$ & 2012 & $\begin{array}{l}2^{\circ} \\
\text { estratégia }\end{array}$ & SIM \\
\hline 11 & $\begin{array}{l}\text { Knowledge transfer, knowledge sharing and } \\
\text { knowledge barriers - three blurry terms in km }\end{array}$ & $\begin{array}{l}\text { Paulin; } \\
\text { Suneson. }\end{array}$ & 2012 & $\begin{array}{l}2^{\circ} \\
\text { estratégia }\end{array}$ & SIM \\
\hline 12 & $\begin{array}{l}\text { Knowledge management: review of the critical success } \\
\text { factors and development of a conceptual classification model }\end{array}$ & $\begin{array}{l}\text { Sedighi; } \\
\text { Zand. }\end{array}$ & 2012 & $\begin{array}{l}2^{\circ} \\
\text { estratégia }\end{array}$ & SIM \\
\hline 13 & $\begin{array}{l}\text { Knowledge management issues in Malaysian organizations: } \\
\text { the perceptions of leaders }\end{array}$ & Moshari. & 2013 & $\begin{array}{l}1^{\mathrm{o}} \\
\text { estratégia }\end{array}$ & SIM \\
\hline 14 & $\begin{array}{l}\text { Knowledge management mapping and gap analysis in } \\
\text { renewable energy: towards a sustainable framework in } \\
\text { developing countries }\end{array}$ & Fadell et al. & 2013 & $\begin{array}{l}1^{\mathrm{o}} \\
\text { estratégia }\end{array}$ & NÃO \\
\hline 15 & $\begin{array}{l}\text { Librarians' perceptions of knowledge management in } \\
\text { developing countries: a case with Indian academic libraries }\end{array}$ & $\begin{array}{l}\text { Nazim; } \\
\text { Mukherjee. }\end{array}$ & 2013 & $\begin{array}{c}1^{\circ} \\
\text { estratégia }\end{array}$ & SIM \\
\hline 16 & $\begin{array}{l}\text { The analysis of the relation of evaluation criteria of } \\
\text { employee's performance with implementation of } \\
\text { knowledge management of Padide Paydar Company }\end{array}$ & Souri et al. & 2013 & $\begin{array}{l}1^{\circ} \\
\text { estratégia }\end{array}$ & SIM \\
\hline 17 & $\begin{array}{l}\text { A three-dimensional model of identifying barriers to } \\
\text { knowledge management }\end{array}$ & $\begin{array}{l}\text { Ujwary- } \\
\text { Gill. }\end{array}$ & 2013 & $\begin{array}{c}1^{\circ} \\
\text { estratégia }\end{array}$ & SIM \\
\hline 18 & $\begin{array}{l}\text { A fuzzy AHP-TOPSIS framework for ranking the solutions } \\
\text { of knowledge management adoption in supply chain to } \\
\text { overcome its barriers }\end{array}$ & $\begin{array}{l}\text { Patil; } \\
\text { Kant. }\end{array}$ & 2013 & $\begin{array}{l}2^{\circ} \\
\text { estratégia }\end{array}$ & SIM \\
\hline 19 & $\begin{array}{l}\text { An empirical investigation into the influence of } \\
\text { knowledge sharing barriers on knowledge sharing and } \\
\text { individual innovation behavior }\end{array}$ & $\begin{array}{l}\text { Yesil; } \\
\text { Hirlak. }\end{array}$ & 2013 & $\begin{array}{l}2^{\circ} \\
\text { estratégia }\end{array}$ & SIM \\
\hline 20 & $\begin{array}{l}\text { Barriers to tacit knowledge retention: an understanding of the } \\
\text { perceptions of the knowledge management of people inside } \\
\text { and outside the organization: original research }\end{array}$ & $\begin{array}{l}\text { Bessick; } \\
\text { Naicker. }\end{array}$ & 2013 & $\begin{array}{l}2^{\circ} \\
\text { estratégia }\end{array}$ & SIM \\
\hline 21 & $\begin{array}{l}\text { Knowledge barriers, knowledge transfer, and innovation } \\
\text { competitive advantage in healthcare settings }\end{array}$ & Sheng et al. & 2013 & $\begin{array}{l}2^{\circ} \\
\text { estratégia }\end{array}$ & SIM \\
\hline 22 & $\begin{array}{l}\text { Knowledge management and the effectiveness of innovation } \\
\text { outcomes: the role of cultural barriers }\end{array}$ & $\begin{array}{l}\text { Rodríguez } \\
\text { et al. }\end{array}$ & 2013 & $\begin{array}{c}2^{\circ} \\
\text { estratégia }\end{array}$ & SIM \\
\hline 23 & $\begin{array}{l}\text { Knowledge sharing barriers in organic growth: A case study } \\
\text { from a software company }\end{array}$ & Kukko. & 2013 & $\begin{array}{l}2^{\circ} \\
\text { estratégia }\end{array}$ & SIM \\
\hline 24 & $\begin{array}{l}\text { An exploration study to detect important barriers for } \\
\text { knowledge management }\end{array}$ & Ghafourian. & 2014 & $\begin{array}{c}2^{\circ} \\
\text { estratégia }\end{array}$ & SIM \\
\hline 25 & $\begin{array}{l}\text { Knowledge management in complementary and processing } \\
\text { industries in Khouzestan Province, Iran }\end{array}$ & $\begin{array}{l}\text { Ommani; } \\
\text { Noorivandi. }\end{array}$ & 2014 & $\begin{array}{c}1^{\mathrm{o}} \\
\text { estratégia }\end{array}$ & SIM \\
\hline 26 & $\begin{array}{l}\text { Knowledge management practice at a Bulgarian bank: a case } \\
\text { study }\end{array}$ & $\begin{array}{l}\text { Shah; } \\
\text { Rahneva; } \\
\text { Ahmed. }\end{array}$ & 2014 & $\begin{array}{l}1^{\circ} \\
\text { estratégia }\end{array}$ & SIM \\
\hline 27 & $\begin{array}{l}\text { Knowledge management practices and performance in } \\
\text { Nigerian universities }\end{array}$ & $\begin{array}{l}\text { Omogeafe; } \\
\text { Friday. }\end{array}$ & 2014 & $\begin{array}{c}1^{\circ} \\
\text { estratégia }\end{array}$ & NÂO \\
\hline
\end{tabular}




\begin{tabular}{|c|c|c|c|c|c|}
\hline 28 & $\begin{array}{l}\text { Knowledge management, entrepreneurial competencies and } \\
\text { organizational development in micro and small enterprises in } \\
\text { rural regions in the state of Yucatan, Mexico }\end{array}$ & $\begin{array}{l}\text { Tanoira; } \\
\text { Valencia. }\end{array}$ & 2014 & $\begin{array}{l}1^{\circ} \\
\text { estratégia }\end{array}$ & NÃO \\
\hline 29 & $\begin{array}{l}\text { Predicting student academic performance: role of } \\
\text { knowledge sharing and outcome expectations }\end{array}$ & $\begin{array}{l}\text { Aslam; } \\
\text { Siddiqi; } \\
\text { Shahzad; } \\
\text { Bajwa. } \\
\end{array}$ & 2014 & $\begin{array}{l}1^{\circ} \\
\text { estratégia }\end{array}$ & NÃO \\
\hline 30 & $\begin{array}{l}\text { A conceptual framework to address barriers to knowledge } \\
\text { management in project-based organizations }\end{array}$ & $\begin{array}{l}\text { Akhavan; } \\
\text { Hosein; } \\
\text { Akhavan. }\end{array}$ & 2014 & $\begin{array}{l}2^{\circ} \\
\text { estratégia }\end{array}$ & SIM \\
\hline 31 & $\begin{array}{l}\text { The barriers of knowledge } \\
\text { generation, storage, distribution and application that impede } \\
\text { learning in gas and petroleum companies }\end{array}$ & $\begin{array}{l}\text { Ranjbarfard } \\
\text { et al. }\end{array}$ & 2014 & $\begin{array}{l}2^{\circ} \\
\text { estratégia }\end{array}$ & SIM \\
\hline 32 & $\begin{array}{l}\text { Knowledge management barriers, practices and maturity } \\
\text { model }\end{array}$ & Oliva & 2014 & $\begin{array}{c}2^{\circ} \\
\text { estratégia }\end{array}$ & SIM \\
\hline 33 & $\begin{array}{l}\text { Knowledge management: barriers to capturing lessons } \\
\text { learned from Australian construction contractor's perspective }\end{array}$ & $\begin{array}{l}\text { Shokri- } \\
\text { Ghasabeh; } \\
\text { Chileshe. }\end{array}$ & 2014 & $\begin{array}{l}2^{\circ} \\
\text { estratégia }\end{array}$ & SIM \\
\hline 34 & $\begin{array}{l}\text { Modeling the metrics of individual, organizational and } \\
\text { technological knowledge sharing barriers: an analytical } \\
\text { network process approach }\end{array}$ & $\begin{array}{l}\text { Sharma; } \\
\text { Singh. }\end{array}$ & 2014 & $\begin{array}{l}2^{\circ} \\
\text { estratégia }\end{array}$ & SIM \\
\hline 35 & $\begin{array}{l}\text { Global social knowledge management: understanding barriers } \\
\text { for global workers utilizing social software. }\end{array}$ & $\begin{array}{l}\text { Pirkkalaine; } \\
\text { Pawlowski. }\end{array}$ & 2014 & $\begin{array}{c}2^{\circ} \\
\text { estratégia }\end{array}$ & SIM \\
\hline 36 & $\begin{array}{l}\text { Knowledge management and new skills, roles \& challenges } \\
\text { for librarians in the ICT world }\end{array}$ & Patel. & 2015 & $\begin{array}{l}1^{\circ} \\
\text { estratégia }\end{array}$ & NÃO \\
\hline 37 & $\begin{array}{l}\text { Knowledge management in Romanian ITC SMEs } \\
\text { (quantitative methods inquires) (small and medium sized } \\
\text { enterprises) }\end{array}$ & Ceptureanu. & 2015 & $\begin{array}{l}1^{\circ} \\
\text { estratégia }\end{array}$ & NÃO \\
\hline 38 & Critical success factors of knowledge management in Albania & $\begin{array}{l}\text { Margilaj; } \\
\text { Bello. }\end{array}$ & 2015 & $\begin{array}{c}1^{\circ} \\
\text { estratégia }\end{array}$ & SIM \\
\hline 39 & $\begin{array}{l}\text { Rethinking knowledge sharing barriers: a content analysis of } \\
103 \text { studies }\end{array}$ & $\begin{array}{l}\text { Cleveland; } \\
\text { Ellis. }\end{array}$ & 2015 & $\begin{array}{c}2^{\circ} \\
\text { estratégia }\end{array}$ & SIM \\
\hline 40 & $\begin{array}{l}\text { Strategic and cultural misalignment: knowledge } \\
\text { sharing barriers in project networks }\end{array}$ & $\begin{array}{l}\text { Solli- } \\
\text { Sæther; } \\
\text { Karlsen; } \\
\text { Oorschot. }\end{array}$ & 2015 & $\begin{array}{l}2^{\circ} \\
\text { estratégia }\end{array}$ & SIM \\
\hline 41 & $\begin{array}{l}\text { The influence of individual characteristics on knowledge } \\
\text { sharing practices, enablers, and barriers in a project } \\
\text { management context }\end{array}$ & $\begin{array}{l}\text { Khalil; } \\
\text { Marouf. }\end{array}$ & 2015 & $\begin{array}{l}2^{\circ} \\
\text { estratégia }\end{array}$ & SIM \\
\hline 42 & $\begin{array}{l}\text { A collaborative methodology for tacit knowledge } \\
\text { management: application to scientific research }\end{array}$ & $\begin{array}{l}\text { Mezghani; } \\
\text { Exposito; } \\
\text { Drira. }\end{array}$ & 2016 & $\begin{array}{l}1^{\circ} \\
\text { estratégia }\end{array}$ & NÃO \\
\hline 43 & $\begin{array}{l}\text { Barriers to knowledge sharing in third sector social care: a } \\
\text { case study }\end{array}$ & $\begin{array}{l}\text { Bloice; } \\
\text { Burnett. }\end{array}$ & 2016 & $\begin{array}{l}2^{\circ} \\
\text { estratégia }\end{array}$ & SIM \\
\hline
\end{tabular}

Fonte: Dados da pesquisa

A terceira etapa baseou-se na identificação das referências utilizadas pelos pesquisadores no desenvolvimento das publicações selecionadas na segunda fase, estabelecendo o critério de seleção através da extração de títulos com termos: barriers, knowledge management, difficulties, failure e limitations.

A condição de seleção dos artigos na terceira fase se baseou na relevância do conteúdo e na quantidade de autores selecionados na segunda fase que utilizaram os artigos como base conceitual. Os artigos selecionados deveriam ter sido utilizados por no mínimo dois autores.

A identificação das referências nas publicações teve como objetivo agregar a literatura utilizada como base conceitual nos artigos selecionados na segunda fase para 
enriquecer o corpo teórico e de análise da pesquisa.

Quadro 2 - Artigos relevantes selecionados na terceira fase

\begin{tabular}{|c|c|c|c|}
\hline Referências & Ano & Fonte & Seleção \\
\hline $\begin{array}{l}\text { BARSON, J. et al. Inter- and intra-organisational } \\
\text { barriers to sharing knowledge in the extended } \\
\text { supply-chain. In: INTERNATIONAL } \\
\text { CONFERENCE ON E-BUSINESS AND E-WORK, } \\
\text { 2000, Madri. Proceeding... Amsterdam: IOS Press, } \\
2000 .\end{array}$ & 2000 & $\begin{array}{l}29 \text { AKHAVAN; HOSEIN; } \\
\text { AKHAVAN (2014) } \\
31 \text { RANJBARFARD et al. (2014) } \\
41 \text { KHALIL; MAROUF (2015) }\end{array}$ & SIM \\
\hline $\begin{array}{l}\text { LUGGER, K. M.; KRAUS, H. Mastering the } \\
\text { human barriers in knowledge management. Journal } \\
\text { of Universal Computer Science, v. } 7 \text {, n. 6, p. 488- } \\
497,2001 .\end{array}$ & 2001 & $\begin{array}{l}\text { 02 LEVY et al (2010) } \\
\text { 03 HONG; SUH; KOO (2011) } \\
10 \text { KHALIL; SHEA (2012) } \\
\text { 17 URWARY (2013) } \\
31 \text { RANJBARFARD et al. (2014) } \\
\end{array}$ & SIM \\
\hline $\begin{array}{l}\text { CANTONI, F.; BELLO, M.; FRIGERIO, C. } \\
\text { Lowering the barriers to knowledge transfer and } \\
\text { dissemination: the Italian cooperative banks } \\
\text { experience. In: EUROPEAN CONFERENCE ON } \\
\text { INFORMATION SYSTEMS, 9., 2001, Bled. } \\
\text { Proceedings... Bled: ECIS, 2001. P. 1-9 }\end{array}$ & 2001 & $\begin{array}{l}03 \text { HONG; SUH; KOO (2011) } \\
31 \text { RANJBARFARD et al. (2014) }\end{array}$ & SIM \\
\hline $\begin{array}{l}\text { DISTERER, G. Individual and Social Barriers to } \\
\text { Knowledge Transfer. In: HAWAII } \\
\text { INTERNATIONAL CONFERENCE ON SYSTEM } \\
\text { SCIENCES, 34., 2001, Hawaii. Proceedings... } \\
\text { Piscataway: IEEE, } 2001\end{array}$ & 2001 & $\begin{array}{l}03 \text { HONG; SUH; KOO (2011) } \\
37 \text { PIRKKALAINE; PAWLOWSKI } \\
(2014)\end{array}$ & SIM \\
\hline $\begin{array}{l}\text { MCDERMOTT, R.; O’ DELL, C. Overcoming } \\
\text { cultural barriers to sharing knowledge. Journal of } \\
\text { Knowledge Management, v. 5, n. 1, p. 76-85, } 2001 .\end{array}$ & 2001 & $\begin{array}{l}03 \text { HONG; SUH; KOO (2011) } \\
\text { 05 KOTHARI et al. (2011) } \\
10 \text { KHALIL; SHEA (2012) } \\
19 \text { YESIL; HIRLAK (2013) } \\
26 \text { SHAN; RAHNEVA; AHMED } \\
\text { (2014) } \\
\text { 31 RANJBARFARD et al. (2014) } \\
\text { 37 PIRKKALAINE; PAWLOWSKI } \\
\text { (2014) } \\
36 \text { SHARMA; SINGH (2014) } \\
45 \text { BLOICE; BURNETT (2016) } \\
13 \text { MOSHARI (2013) }\end{array}$ & SIM \\
\hline $\begin{array}{l}\text { BUREŠ, V. Cultural barriers in knowledge sharing. } \\
\text { E+M Ekonomics and Management, v.6, p.57-62, } \\
2003\end{array}$ & 2003 & $\begin{array}{l}\text { 03 HONG; SUH; KOO (2011) } \\
10 \text { KHALIL; SHEA (2012) } \\
\text { 22 RODRIGUEZ et al. (2013) } \\
\text { 31 RANJBARFARD et al. (2014) } \\
\text { 37-PIRKKALAINE; PAWLOWSKI } \\
\text { (2014) }\end{array}$ & SIM \\
\hline $\begin{array}{l}\text { RIEGE, A. Three-dozen knowledge-sharing barriers } \\
\text { managers must consider. Journal of Knowledge } \\
\text { Management, v. } 9 \text {, n. } 3 \text {, p. } 18-35,2005 \text {. }\end{array}$ & 2005 & $\begin{array}{l}\text { 08 LIN; WU; YEN (2012) } \\
10 \text { KHALIL; SHEA (2012) } \\
11 \text { PAULIN; SINESON (2012) } \\
17 \text { URWARY (2013) } \\
19 \text { YESIL; HIRLAK (2013) } \\
20 \text { BESSICK; NAICKER (2013) } \\
22 \text { RODRIGUEZ et al. (2013) } \\
24 \text { GHAFOURIAN (2014) } \\
29 \text { AKHAVAN; HOSEIN; } \\
\text { AKHAVAN (2014) } \\
31 \text { RANJBARFARD et al. (2014) } \\
35 \text { OLIVA (2014) }\end{array}$ & SIM \\
\hline
\end{tabular}




\begin{tabular}{|c|c|c|c|}
\hline & & $\begin{array}{l}37 \text { PIRKKALAINE; PAWLOWSKI } \\
(2014) \\
\text { 41 CLEVELAND; ELLIS (2015) } \\
41 \text { KHALIL; MAROUF }(2015) \\
45 \text { BLOICE; BURNETT (2016) } \\
36 \text { SHARMA; SINGH }(2014)\end{array}$ & \\
\hline $\begin{array}{l}\text { SVETLANA, S. An investigation of critical barriers } \\
\text { to effective knowledge management. Social } \\
\text { Sciences/Socialiniai Mokslai, v. 58, n. } 4,2007 .\end{array}$ & 2007 & $\begin{array}{l}10 \text { KHALIL; SHEA (2012) } \\
22 \text { RODRIGUEZ et al. (2013) } \\
31 \text { RANJBARFARD et al. (2014) } \\
\text { 41 CLEVELAND; ELLIS (2015) }\end{array}$ & SIM \\
\hline
\end{tabular}

Fonte: elaborado pelo autor

\section{BARREIRAS À GESTÃO DO CONHECIMENTO}

Um desafio fundamental para as organizações é como incentivar o compartilhamento de conhecimento internamente entre os funcionários (HONG; SUH; KOO, 2011). Desde o momento em que as pessoas se encontram relutantes para compartilhar seus conhecimentos é criada uma série de barreiras para a gestão do conhecimento. Para organizações, as barreiras da gestão do conhecimento são os desafios enfrentados no seu desenvolvimento e sua implantação, onde envolve as pessoas a participarem das atividades do gerenciamento efetivo do conhecimento (MARGILAJ; BELLO, 2015).

A internalização das experiências vivenciadas pelos indivíduos, assim como as posteriores reflexões é um processo demorado e complexo Nas organizações é comum vivenciar a cultura do individualismo, onde o objetivo é ser "útil" ou se destacar com características técnicas diferenciadas. Para alcançar tal objetivo, os indivíduos acumulam o conhecimento individual, acarretando na formação de um "campo de batalha" entre os funcionários (HERRGARD, 2000).

$\mathrm{Na}$ avaliação e implementação de um sistema de gestão do conhecimento em uma organização, é importante compreender como as diferentes barreiras ao fluxo de conhecimento afetam a evolução organizacional (LIN; WU; YEN, 2012). Porém as organizações não possuem comportamentos proativos, as soluções e desenvolvimento de estratégias para enfrentar barreiras relacionadas ao gerenciamento do conhecimento geralmente ocorrem apenas após a existência dos problemas (PIRKKALAINEN; PAWLOWSKI 2014).

Nas organizações, elementos como maus hábitos, técnicas e comportamento obsoleto podem ser difundidos na cultura organizacional (HERRGARD, 2000), inibindo a produtividade e eficácia nos sistemas de gestão (MCDERMOTT; O'DELL, 2001) e o próprio compartilhamento do conhecimento (HONG; SUH; KOO, 2000).

\section{ANÁLISE DOS DADOS}

Analisando os 44 artigos selecionados, foram identificadas 49 categorias em que os autores organizaram as barreiras e desafios à gestão do conhecimento evidenciadas em seus estudos de casos ou revisões de literatura.

Apesar da quantidade elevada de categorias, percebe-se a concentração de utilização de três categorias específicas (individual, organizacional e tecnológica), onde os autores em suas pesquisas, categorizaram as barreiras e desafios à gestão do conhecimento. A categoria de barreiras individuais foi citada em 15 artigos, a categoria de barreiras organizacionais foi citada em 21 artigos e a categoria de barreiras tecnológicas foi citada em 15 artigos.

Em alguns casos, nota-se que os autores utilizaram elementos comumente identificado como barreiras, formalizando-as em categoria/classificação.

Quadro 3 - Categorização das barreiras (relação de autores)

\begin{tabular}{|c|c|c|c|}
\hline Categorias de barreiras & Citações & Autores & Ano \\
\hline \multirow{3}{*}{ Individual } & \multirow{3}{*}{15} & DISTERER & 2001 \\
\hline & & LUGGER; KRAUS & 2001 \\
\hline & & BURES & 2003 \\
\hline
\end{tabular}




\begin{tabular}{|c|c|c|c|}
\hline & & RIEGE & 2005 \\
\hline & & SVETLANA & 2007 \\
\hline & & HONG; SUH; KOO & 2011 \\
\hline & & KOTHARI et al. & 2011 \\
\hline & & KUKKO & 2013 \\
\hline & & PATIL; KANT & 2013 \\
\hline & & UJWARY-GILL & 2013 \\
\hline & & SHARMA; SING & 2014 \\
\hline & & AKHAVAN; ZEHEDI; HOSEIN & 2014 \\
\hline & & GHAFOURIAN & 2014 \\
\hline & & MAROUF; KHALIL & 2015 \\
\hline & & BLOICE; BURNETT & 2016 \\
\hline \multirow{4}{*}{ Social } & \multirow{4}{*}{4} & BURES & 2003 \\
\hline & & DISTERER & 2001 \\
\hline & & PIRKKALAINEN; PAWLOWSKI & 2014 \\
\hline & & UJWARY-GILL & 2013 \\
\hline \multirow{2}{*}{ Pessoas } & \multirow{2}{*}{2} & BARSON et al. & 2000 \\
\hline & & RANJBARFARD et al. & 2014 \\
\hline Humana & 1 & OLIVA & 2014 \\
\hline \multirow{21}{*}{ Organizacionais } & \multirow{21}{*}{21} & BARSON et al. & 2000 \\
\hline & & LUGGER; KRAUS & 2001 \\
\hline & & RIEGE & 2005 \\
\hline & & SVETLANA & 2007 \\
\hline & & MCGURN & 2011 \\
\hline & & KOTHARI et al. & 2011 \\
\hline & & SEDIGHI; ZAND & 2012 \\
\hline & & KUKKO & 2013 \\
\hline & & YESIL; HIRLAK & 2013 \\
\hline & & PATIL; KANT & 2013 \\
\hline & & UJWARY-GILL & 2013 \\
\hline & & MOSHARI & 2013 \\
\hline & & HONG; SUH; KOO & 2013 \\
\hline & & PIRKKALAINEN; PAWLOWSKI & 2014 \\
\hline & & SHARMA; SING & 2014 \\
\hline & & OLIVA & 2014 \\
\hline & & AKHAVAN; ZEHEDI; HOSEIN & 2014 \\
\hline & & GHAFOURIAN & 2014 \\
\hline & & MAROUF; KHALIL & 2015 \\
\hline & & MARGILAJ; BELLO & 2015 \\
\hline & & BLOICE; BURNETT & 2016 \\
\hline \multirow{6}{*}{ Cultural } & \multirow{6}{*}{6} & VAZQUEZ; FOURNIER; FLORES & 2009 \\
\hline & & KOTHARI et al. & 2011 \\
\hline & & RODRÍGUEZ et al. & 2013 \\
\hline & & PATIL; KANT & 2013 \\
\hline & & GHAFOURIAN & 2014 \\
\hline & & $\begin{array}{l}\text { SOLLI-SÆTHER; KARLSEN; } \\
\text { OORSCHOT }\end{array}$ & 2015 \\
\hline \multirow{9}{*}{ Tecnológicas } & \multirow{9}{*}{15} & SVETLANA & 2007 \\
\hline & & RIEGE & 2005 \\
\hline & & BARSON et al. & 2000 \\
\hline & & MCGURN & 2011 \\
\hline & & KUKKO & 2013 \\
\hline & & YESIL; HIRLAK & 2013 \\
\hline & & PATIL; KANT & 2013 \\
\hline & & MOSHARI & 2013 \\
\hline & & PIRKKALAINEN; PAWLOWSKI & 2014 \\
\hline
\end{tabular}




\begin{tabular}{|c|c|c|c|}
\hline & & SHARMA; SING & 2014 \\
\hline & & RANJBARFARD et al. & 2014 \\
\hline & & AKHAVAN; ZEHEDI; HOSEIN & 2014 \\
\hline & & MAROUF; KHALIL & 2015 \\
\hline & & MARGILAJ; BELLO & 2015 \\
\hline & & BLOICE; BURNETT & 2016 \\
\hline \multirow{4}{*}{ Sem categoria } & \multirow{4}{*}{4} & MCDERMOTT; O'DELL & 2001 \\
\hline & & CANTONI; BELLO; FRIGERIO & 2001 \\
\hline & & SVETLANA & 2007 \\
\hline & & KHALIL; SHEA & 2012 \\
\hline \multirow{2}{*}{ Relacionadas ao gerenciamento de projetos } & \multirow{2}{*}{2} & SHAH; RAHNEVA; AHMED & 2014 \\
\hline & & AKHAVAN; ZEHEDI; HOSEIN & 2014 \\
\hline $\begin{array}{l}\text { Entre projetos da gestão do conhecimento em } \\
\text { organizações baseados em projetos }\end{array}$ & 1 & AKHAVAN; ZEHEDI; HOSEIN & 2014 \\
\hline Desalinhamento estratégico & 1 & $\begin{array}{l}\text { SOLLI-SÆTHER; KARLSEN; } \\
\text { OORSCHOT }\end{array}$ & 2015 \\
\hline Proteção de conhecimento & \multirow{3}{*}{1} & \multirow{3}{*}{ CLEVELAND; ELLIS } & \multirow{3}{*}{2015} \\
\hline Comportamento de busca do conhecimento & & & \\
\hline Inibidores da contribuição do conhecimento & & & \\
\hline Cruzadas & 1 & BARSON et al. & 2000 \\
\hline Relacionadas à natureza do conhecimento & 1 & SVETLANA & 2007 \\
\hline Relacionadas a processos / organização & \multirow{3}{*}{1} & \multirow{3}{*}{ RANJBARFARD et al. } & \multirow{3}{*}{2014} \\
\hline Barreiras relacionadas ao meio ambiente & & & \\
\hline Relacionadas às características do conhecimento & & & \\
\hline $\begin{array}{l}\text { Variáveis que afetam diretamente a gestão do } \\
\text { conhecimento }\end{array}$ & 1 & OMMANI; NOORIVANDI & 2014 \\
\hline Para capturar lições aprendidas & 1 & SHOKRI-GHASABEH; CHILESHE & 2014 \\
\hline Liderança & 1 & MARGILAJ; BELLO & 2015 \\
\hline Características do trabalho & \multirow{3}{*}{1} & \multirow{3}{*}{ BESSICK; NAICKER } & \multirow{3}{*}{2013} \\
\hline Satisfação do trabalho & & & \\
\hline Gerenciamento de talentos & & & \\
\hline Barreiras técnicas & 1 & UJWARY-GILL & 2013 \\
\hline Criação de conhecimento & \multirow{4}{*}{1} & \multirow{4}{*}{ SOURI et al. } & \multirow{4}{*}{2013} \\
\hline Manutenção do conhecimento & & & \\
\hline Transição do conhecimento & & & \\
\hline Aplicação do conhecimento & & & \\
\hline Incorporação da gestão do conhecimento & \multirow{2}{*}{1} & \multirow{2}{*}{ NAZIM; MUKHERJEE } & \multirow{2}{*}{2013} \\
\hline Aplicações da prática da gestão do conhecimento & & & \\
\hline Treinamento de funcionário & \multirow{3}{*}{1} & \multirow{3}{*}{ MOSHARI } & \multirow{3}{*}{2013} \\
\hline Gestão & & & \\
\hline $\begin{array}{l}\text { Financeiro } \\
\end{array}$ & & & \\
\hline Plataformas de compartilhamento do conhecimento & & & \\
\hline Cooperação & 1 & NDLELA & 2012 \\
\hline Comunicação & 1 & NDLELA & 2012 \\
\hline Fatores epistémicos & & & \\
\hline Receptor do conhecimento & & & \\
\hline Fatores contextuais & 1 & LIN $\cdot \mathrm{WU} \cdot \mathrm{YEN}$ & 2012 \\
\hline Mecanismos & & & \\
\hline $\begin{array}{l}\text { Fonte de conhecimento } \\
\end{array}$ & & & \\
\hline $\begin{array}{l}\text { "Ninguém pode usar meu conhecimento" / medo de } \\
\text { ser considerado tolo }\end{array}$ & & & \\
\hline Conhecimento como poder & 1 & LILLEOERE: HANSEN & 2011 \\
\hline Sem proximidade física com os colegas & 1 & LILLEUERE; HAINSEN & 2011 \\
\hline Falta de apreciação e atenção & & & \\
\hline "Não sei quem sabe" & & & \\
\hline
\end{tabular}

Fonte: Dados da pesquisa 
Após o trabalho de análise desenvolvido é possível afirmar a existência de barreiras que são recorrentes aos variados contextos organizacionais.

Foi possível perceber a recorrência de barreiras nas três categorias adotadas para a construção e organização da lista geral.

$\mathrm{Na}$ categoria individual, a falta de confiança nas pessoas pelo potencial de abuso ou pelo crédito injusto foi a barreira mais evidenciada, elevando a importância da confiança mútua entre os indivíduos da organização para que a gestão do conhecimento produza os resultados desejados.

$\mathrm{Na}$ categoria organizacional, a falta de apoio, suporte e envolvimento ativo e contínuo da alta administração (gestão) e falta de liderança se destacam e direcionam o nível de qualidade em iniciativas de gestão do conhecimento de acordo com a participação direta dos gestores e da alta administração nos processos de estruturação e coordenação dos programas aplicados nas organizações.

$\mathrm{Na}$ categoria tecnológica a falta de tecnologias que atendam às necessidades de compartilhamento foi considerada a barreira mais recorrente. Para as organizações, a seleção de tecnologias adequadas para programas de gestão do conhecimento é um grande desafio, pois envolve o equilíbrio de variados elementos, como, falta de recurso, configurações e adequações ao propósito escolhido, reações e adaptações por parte dos funcionários, treinamentos, entre outros elementos.

De acordo com os resultados da pesquisa, pode-se considerar que o processo de implantação da gestão do conhecimento e suas práticas, conduzem a determinados contextos de recorrentes barreiras que estão naturalmente presentes pela complexidade de gerir $\mathrm{o}$ conhecimento. A natureza humana contribui com variadas motivações, ambições e sentimentos que originam barreiras focadas nos receios, medos e falta de confiança. No aspecto organizacional, a própria deficiência da gestão e coordenação, e falta de recursos, conduz ao insucesso de programas de gestão do conhecimento aplicados nas rotinas e atividades do dia a dia.

Apesar das recorrências das barreiras serem originadas por condições e consequências de práticas comuns presentes na implantação da gestão do conhecimento, cada organização tem sua particularidade dentro do contexto as quais estão inseridas, sofrendo influências específicas do ambiente interno e externo. Essas influências impactam diretamente no estado de aprendizagem e compartilhamento do conhecimento. Nessas condições, mesmo que em menor escala, são criadas barreiras que são exclusivas ao contexto da organização.

Quadro 4 - Identificação das principais barreiras à gestão do conhecimento e suas recorrências

\begin{tabular}{|l|l|c|}
\hline Categorias & \multicolumn{1}{|c|}{ Barreiras } & Citações \\
\hline \multirow{5}{*}{ Individual } & Falta de confiança nas pessoas pelo potencial abuso ou pelo crédito injusto & 17 \\
\cline { 2 - 3 } & $\begin{array}{l}\text { Falta de tempo para compartilhar ou se dedicar nas atividades da gestão do } \\
\text { conhecimento }\end{array}$ & 15 \\
\cline { 2 - 3 } & Pobre comunicação verbal e escrita & 13 \\
\cline { 2 - 3 } & $\begin{array}{l}\text { Diferenças de cultura nacional ou origem étnica; valores e crenças associadas } \\
\text { (incluindo a linguagem). }\end{array}$ & 9 \\
\cline { 2 - 3 } Citações: 15 & Medo de reduzir a segurança no trabalho, se tornando substituível & 9 \\
\cline { 2 - 3 } & Falta de encorajamento e incentivos & 8 \\
\cline { 2 - 3 } & $\begin{array}{l}\text { Baixa valorização e consciência do benefício do conhecimento possuído para os } \\
\text { outros. }\end{array}$ & 9 \\
\cline { 2 - 3 } & Medo de desistir do poder, da autoridade e exclusividade & 6 \\
\cline { 2 - 3 } & Falta de credibilidade do conhecimento devido à fonte & 6 \\
\cline { 2 - 3 } & $\begin{array}{l}\text { Medo de assumir a propriedade intelectual devido ao receio de receber críticas, } \\
\text { carga de trabalho adicional ou opiniões contrárias }\end{array}$ & 5 \\
\cline { 2 - 3 } & Diferenças nos níveis de experiência profissional & 5 \\
\cline { 2 - 3 } & Falta de motivação & 5 \\
\cline { 2 - 3 } & Falta de redes sociais & 5 \\
\cline { 2 - 3 } & Individualismo, sem cultura de equipe & 5 \\
\cline { 2 - 3 } & Incerteza sobre o valor do conhecimento possuído & 5 \\
\hline
\end{tabular}




\begin{tabular}{|c|c|c|}
\hline \multirow{6}{*}{ Organizacionais: } & $\begin{array}{l}\text { Falta de apoio, suporte e envolvimento ativo e contínuo da alta administração - } \\
\text { gestão }\end{array}$ & 15 \\
\hline & Falta de liderança & 10 \\
\hline & $\begin{array}{l}\text { Espaços insuficientes formais e informais para colaborar e refletir e gerar (novos) } \\
\text { conhecimento. }\end{array}$ & 10 \\
\hline & Tempo e recursos insuficientes para atividades de gestão do conhecimento & 10 \\
\hline & Falta de treinamento & 9 \\
\hline & Falta de infraestruturas apoiadoras adequadas para a prática de compartilhamento & 9 \\
\hline \multirow[t]{5}{*}{ Citações: 21} & $\begin{array}{l}\text { Burocracia e hierarquias rígidas que inibem ou retarda a maioria das práticas de } \\
\text { compartilhamento }\end{array}$ & 9 \\
\hline & Competição interna elevada & 8 \\
\hline & Medo ou inércia para mudar, resistência contra a mudança & 8 \\
\hline & Sistemas de motivação e recompensa insuficientes & 7 \\
\hline & Distância geográfica & 7 \\
\hline \multirow{5}{*}{ Tecnológica: } & Falta de tecnologias que atendam às necessidades de compartilhamento & 10 \\
\hline & Falta de familiaridade, habilidades e experiência com o novo sistema de TI & 9 \\
\hline & $\begin{array}{l}\text { Falta de gestão na comunicação das vantagens e valores das práticas de } \\
\text { compartilhamento do conhecimento }\end{array}$ & 7 \\
\hline & Expectativas irrealistas dos funcionários sobre o potencial da tecnologia & 7 \\
\hline & $\begin{array}{l}\text { Práticas de compartilhamento restritos ao ambiente físico e layout das áreas de } \\
\text { trabalho }\end{array}$ & 6 \\
\hline \multirow{3}{*}{ Citações: 15} & Incompatibilidade entre os requisitos das pessoas e os sistemas de TI & 6 \\
\hline & Falta de treinamento sobre familiarização de funcionários de novos sistemas de TI & 6 \\
\hline & $\begin{array}{l}\text { Falta de integração da estratégia e iniciativas de compartilhamento das metas e } \\
\text { objetivos da empresa }\end{array}$ & 5 \\
\hline
\end{tabular}

Fonte: dados da pesquisa

\section{SUPERAÇÃO DAS BARREIRAS}

Na implantação da gestão do conhecimento são necessárias transformações na cultura corporativa, soluções tecnológicas e procedimentos de trabalho existentes. A interação entre sistemas sociais e tecnológicos deve ser compreendida com clareza, com restrições mínimas de tempo e espaço. Mas são os indivíduos que definem o significado da informação com suas interpretações dos contextos e situações (BURES, 2003).

Antes de implementar um programa de gestão do conhecimento é necessário identificar os problemas, barreiras e fatores críticos reais e potenciais que estão presentes na organização que impeçam o sucesso dessa iniciativa. Tendo conhecimento dos motivos da falha no gerenciamento do conhecimento, é possível investigar as razões pelas quais o gerenciamento do conhecimento não é bemsucedido, oferecendo uma percepção da estrutura mais adequada para a implementação de iniciativas de gerenciamento de conhecimento em uma organização (SVETLANA, 2007, MARGILAJ; BELLO, 2015, LEVY et al., 2010).

$O$ desafio principal não se concentra em tratamento de informações ou adequações de recursos tecnológicos, mas nas barreiras culturais e a dificuldade da mudança de comportamento (DISTERER, 2001). Em muitas organizações, é necessária uma grande mudança cultural para mudar as atitudes e o comportamento dos funcionários, de modo que compartilhem seus conhecimentos e percepções de forma voluntária e consistente (LEVY et al., 2010).

O compartilhamento de conhecimentos para os indivíduos e organizações só terá valor real de acordo com o nível de necessidade e se os conhecimentos úteis são recebidos, aceitos e aplicados pelos mesmos (RIEGE, 2005), envolvendo uma atitude de preocupação e confiança entre os membros da organização (DISTERER, 2001).

Não existe uma fórmula geral para uma estratégia de gestão do conhecimento que resulte em uma melhor eficácia para todas as empresas e não há ferramentas práticas padronizadas que irá garantir o total sucesso. As organizações precisam se esforçar para que o conhecimento chegue para as pessoas no momento e no tempo adequado (RIEGE, 2005), proporcionando o aumento da capacidade de reação e compreensão dos ambientes externos e da livre transferência e 
fluxo de informações (SEDIGHI; ZAND, 2012).

O desenvolvimento de um ambiente de compartilhamento e aprendizagem não se resume a altos investimentos financeiros, as organizações já utilizam redes de compartilhamento formais e informais que podem ser expandidas visando adaptações e melhorias, baseadas na análise da lacuna entre o ideal e o estado atual de compartilhamento e valores teoricamente estabelecido e praticados (RIEGE, 2005).

As metas e as estratégias de compartilhamento devem centrar-se em três fatores principais (RIEGE, 2005):

- Motivação e estimulo dos empregados individuais no processo de captura, disseminação, transferência e aplicação dos conhecimentos úteis existentes e recentemente gerados, especialmente o conhecimento tácito;

- Abrir estruturas organizacionais que facilitem fluxos, processos e recursos transparentes de conhecimento que proporcionem uma cultura organizacional de aprendizado contínuo, comunicação clara dos objetivos e estratégia da empresa, ligando práticas e benefícios de compartilhamento de conhecimento;

- Tecnologia moderna que integre os mecanismos e sistemas, proporcionando assim uma plataforma de compartilhamento adequada, acessível a todos os que necessitam de conhecimentos de diversas fontes internas e externas.

\section{CONSIDERAÇÕES FINAIS}

As principais barreiras que estão presentes nos processos de gerenciamento do conhecimento foram apresentadas em diferentes abordagens através do estudo de pesquisas selecionadas sobre $\mathrm{o}$ tema. Amplamente, as categorias de barreiras possuem similaridades de agrupamentos, concentrando-se em sua maioria em fatores individuais e organizacionais, e se relacionam com o impacto presente na utilização dos recursos e ferramentas tecnológicas.

As barreiras à gestão do conhecimento são provenientes de várias fontes humanas e organizacionais, porém após esse estudo, visando o sucesso de iniciativas de gestão do conhecimento, fica evidenciado para esse fim, a importância da confiança mútua entre os indivíduos, a participação e apoio dos gestores nos processos de estruturação e coordenação dos programas e no equilíbrio da seleção e utilização de tecnologias disponíveis de acordo com os recursos e realidade da organização.

Uma implantação bem-sucedida da gestão do conhecimento gera benefícios, permitindo que as organizações tenham um diferencial estratégico na criação, produção, coordenação de processos e principalmente deixa um ambiente propício onde os indivíduos estejam motivados a serem eficientes tecnicamente, compartilhando seus conhecimentos e promovendo o crescimento contínuo organizacional. Porém, com os resultados da pesquisa, existem diversos elementos limitantes na aplicação da gestão do conhecimento nas organizações, sendo necessário desenvolver um planejamento bem elaborado de acordo com os contextos organizacionais, levando em consideração os possíveis fatores que possam impedir a implantação e implementação da gestão do conhecimento. 


\section{CHALLENGES AND BARRIERS TO THE IMPLANTATION OF KNOWLEDGE MANAGEMENT IN ORGANIZATIONS}

Abstract

The present study, through a systematic review of the literature, proposed analyzing scientific articles that approach the difficulties of implementing or managing knowledge management programs. The objective of the research was to study the main categories of barriers to the implementation of knowledge management that were reported by the authors in their research, in order to verify the existence of barriers considered recurrent to a general organizational context or the existence of specific barriers, limited only to the analyzed environment (case studies). The bibliographic research was performed in the Capes Journals Portal database. In the process of retrieval of articles, the search period was defined between the years of 2009 to 2016. A total of 44 articles with the theme proposed for the study were analyzed. The results indicated the concentration of use of three specific categories (individual, organizational and technological). It was possible to identify that, for the most part, barriers to knowledge management are considered recurrent to a general organizational context. Lack of confidence, the lack of support from top management, and the lack of technologies that meet sharing needs were considered the most recurrent barriers.

Keywords: Knowledge Management, Barriers, Challenges, Implantation, Organizations.

\section{REFERÊNCIAS}

BARSON, J. et al. Inter- and intraorganisational barriers to sharing knowledge in the extended supply-chain. In:

INTERNATIONAL CONFERENCE ON EBUSINESS AND E-WORK, 2000, Madri.

Proceeding... Amsterdam: IOS Press, 2000.

BEM, R. M.; PRADO, M. L.; DELFINO, N. Desafios à implantação da gestão do conhecimento: a questão cultural nas organizações públicas federais brasileiras.

Rev. Digit. Bibliotecon. Cienc. Inf., v. 11, n. 2, p.125-135, maio/ago, 2013.

BUREŠ, V. Cultural barriers in knowledge sharing. E+M Ekonomics and Management, v.6, p.57-62, 2003.

CHIAVENATO, I. Introdução à teoria geral da administração. 7. ed. São Paulo: Campus, 2003. $634 \mathrm{p}$.

\section{CHOO, C. W. A organização do}

conhecimento: como as organizações usam a informação para criar significado, construir conhecimento e tomar decisões. 2. ed. São Paulo: Senac, 2006. 425 p.

DISTERER, G. Individual and Social Barriers to Knowledge Transfer. In: HAWAII INTERNATIONAL CONFERENCE ON
SYSTEM SCIENCES, 34., 2001, Hawaii. Proceedings... Piscataway: IEEE, 2001.

EIRIZ, V.; SIMÕES, J.; GONÇALVES, M. Obstáculos à gestão do conhecimento nas escolas de gestão e economia do ensino superior público em Portugal.

Comportamento Organizacional e Gestão, v. 13, n. 2, p. 153-167, 2007.

GALVÃO, T. F.; PEREIRA, M. G. P. Revisões sistemáticas da literatura: passos para sua elaboração. Epidemiol. Serv. Saúde, v. 23, n. 1, p. 183-184, jan./mar. 2014.

GARVIN, D. A. Leaning in action: a guide to putting the learning organization to works. Boston: Harvard Business School Press, 2000.

GHAFOURIAN, J. An exploration study to detect important barriers for knowledge management. Management Science Letters, n. 4, p. 25-30, 2014.

GRANT, M. G.; BROTH, A. A typology of reviews: an analysis of 14 review types and associated methodologies. Health Information and Libraries Journal, v. 26, p. 91-108, 2009.

HARD, H.; LINDKVIST, B. Knowledge creation and transfer in a global technology base organization. In: MBA LUISS 
SEMINAR, Rome, 2000. Proceedings... Rome: s.n, 2000.

HONG, D.; SUH, E.; KOO, C. Developing strategies for overcoming barriers to knowledge sharing based on conversational knowledge management: a case study of a financial company.

Expert Systems with Applications, v. 38, p. 14417-14427, 2011.

KUKKO, M. Knowledge sharing barriers in organic growth: a case study from a software company. Journal of High Technology

Management Research, v.24, p. 18-29, 2013.

LEVY et al. Uncovering cultural perceptions and barriers during knowledge audit. Journal of Knowledge Management, v.14, n. 1, p.114-127, 2010.

LIN, C.; WU, J.-C.; YEN, D. C. Exploring barriers to knowledge flow at different knowledge management maturity stages.

Information \& Management, v. 49, p. 10-23, 2012.

LOPES, P. C. B; STADLER, C. C.;

KOVALESKI, J. L. Gestão organizacional. UEPG Ci. Hum., Ci Soc. Apl., Ling., Letras e Artes, v.11, n. 1, p.51-57, 2003.

MARGILAJ, E.; BELLO, K. Critical success factors of knowledge management in Albania business organizations. European Journal of Research and Reflection in Management Sciences, v. 3, n. 2, 2015

MARQUES JÚNIOR, E.; ALBINO, J. P. Gestão do conhecimento e recursos humanos em bibliotecas universitárias e portuguesas. Nova Série, v.7, n.1, p. 74-89, jan./jun. 2011.

MCDERMOTT, R.; O’ DELL, C. Overcoming cultural barriers to sharing knowledge.

Journal of Knowledge Management, v. 5, n. 1, p. 76-85, 2001.

MUNIZ JÚNIOR, J. et al. Gestão do conhecimento em sistemas produtivos. In: OLIVEIRA, V. F. D.; CAVENAGHI, V. et al (Ed.). Tópicos emergentes e desafios metodológicos em engenharia de produção: casos, experiências e proposições. Rio de Janeiro: Abepro, 2009. v.2.
NONAKA, I.; TAKEUCHI, H. Gestão do conhecimento. Porto Alegre: Bookman, 2008. $319 \mathrm{p}$. PIRKKALAINEN, H.; PAWLOWSKI, J. M. Global social knowledge management: understanding barriers for global workers utilizing social software. Computers in Human Behavior, v. 30, p. 637-647, 2014.

QUEL, L. F. Gestão de conhecimento: e os desafios da complexidade nas organizações. São Paulo: Saraiva, 2006.

PROBST. G.; RAUB, S.; ROMHARDT, K. Gestão do conhecimento: os elementos construtivos do sucesso. Porto Alegre: Bookman, 2002.

RIEGE, A. Three-dozen knowledge-sharing barriers managers must consider. Journal of Knowledge Management, v. 9, n. 3, p. 18-35, 2005.

RUNTE, G. I. B. C. Gestão do conhecimento: os desafios da implantação de um modelo integrado: o caso ANS. 2011. 146 f. Dissertação (Mestrado em Administração Pública) - Fundação Getúlio Vargas, Rio de Janeiro. 2016.

SEDIGHI, M.; ZAND, F. Knowledge management: review of the critical success factors and development of a conceptual classification model. In: INTERNATIONAL CONFERENCE ON ICT AND KNOWLEDGE ENGINEERING, 10., 2012, Bangkok. Proceedings... Piscataway: IEEE, 2012.

SVETLANA, S. An investigation of critical barriers to effective knowledge management. Social Sciences/Socialiniai Mokslai, v. 58, n. 4, 2007.

TERRA, J. C. C. Gestão do conhecimento: o grande desafio empresarial. São Paulo: Terra Fórum, 2010.

TRANFIELD, D.; DENYER, D.; SMART, P. Towards a methodology for developing evidence-informed management knowledge by means of systematic review. British Journal of Management, v. 14, n. 3, p. 207-222, 2003. 
TREHAN, R. Knowledge management: a business perspective. Maastricht:

Hospitalutynet, 2005.

YESIL, S.; HIRLAK, B. An empirical

investigation into the influence of knowledge sharing barriers on knowledge sharing and individual innovation behavior. International Journal of Knowledge Management, v. 9, n. 2,2013 . 